\title{
Constructive and Destructive Interplay between Piezoelectricity and Flexoelectricity in Flexural Sensors and Actuators
}

\author{
Amir Abdollahi Irene Arias* \\ Laboratori de Cálcul Numéric \\ Departament de Matemática Aplicada III \\ Universitat Politécnica de Cataluña \\ Jordi Girona 1-3 E-08034 Barcelona, Spain \\ Email: irene.arias@upc.edu
}

Flexoelectricity is an electromechanical effect coupling polarization to strain gradients. It fundamentally differs from piezoelectricity because of its size-dependence and symmetry. Flexoelectricity is generally perceived as a small effect noticeable only at the nanoscale. Since ferroelectric ceramics have a particularly high flexoelectric coefficient, however, it may play a significant role as piezoelectric transducers shrink to the sub-micrometer scale. We examine this issue with a continuum model self-consistently treating piezo- and flexoelectricity. We show that in piezoelectric device configurations that induce strain gradients and at small but technologically relevant scales, the electromechanical coupling may be dominated by flexoelectricity. More importantly, depending on the device design flexoelectricity may enhance or reduce the effective piezoelectric effect. Focusing on bimorph configurations, we show that configurations that are equivalent at large scales exhibit dramatically different behavior for thicknesses below $100 \mathrm{~nm}$ for typical piezoelectric materials. Our results suggest flexoelectric-aware designs for small-scale piezoelectric bimorph transducers.

\section{Introduction}

Electromechanical coupling mechanisms in materials allow us to sense, actuate, and generally transform mechanical energy into electrical energy and vice versa. Piezoelectricity is one such mechanism, by which electric charges accumulate in response to strain. Another related mechanism is flexoelectricity, which refers to a two-way coupling between electric polarization and strain gradients, rather than strain as in piezoelectricity, or between polarization gradients and strain [1-3]. Because piezoelectricity is scale invariant but flexoelectricity is not, the ratio between flexo- and piezoelectric coefficients defines a length-scale of strain gradient relaxation, below which flexoelectricity is a

${ }^{*}$ Corresponding author. stronger effect $[4,5]$. This lengthscale ranges between $10 \mathrm{~s}$ of nanometers to a micron for common piezoelectrics. As a result, flexoelectricity may be a more efficient mechanism for sensing $[6,7]$ or energy harvesting [8-10] at small scales. Being in principle a universal property for all dielectric materials, flexoelectricity may broaden the class of materials for electromechanical transduction [11, 12], including soft materials $[13,14]$. Flexoelectricity may enable electromechanical transduction at high temperatures, where ferroelectric perovskites are in the paraelectric phase but exhibit large flexoelectric coefficients $[3,15]$. Furthermore, flexoelectric transduction is devoid of aging due to depoling.

Flexoelectricity is commonly neglected in designing or analyzing electromechanical transducers. Yet, piezoelectric thin-film devices are reaching thicknesses of $10 \mathrm{~s}$ to $100 \mathrm{~s} \mathrm{~nm}$, using either PZT and AIN ceramics $[16,17]$ or PVDF polymers [18], which suggests that flexoelectric effects may become significant. Furthermore, since piezo- and flexoelectricity are fundamentally different in terms of symmetry, it is not clear under which circumstances these two effects can cancel each other or act in concert. Here, we examine the interplay between piezo- and flexoelectricity and the resulting electromechanical performance in flexural piezoelectric transducers using numerical computations based on a selfconsistent continuum theory accounting for these two effects $[4,5]$. We focus on bimorph cantilever beams, which are among the most common configurations for piezoelectric transduction $[19,20]$. Applications of bimorphs include ultrasonic motors, laser beam deflectors, fans for heat management in microelectronics, numeric displays, filters, accelerometers, optical choppers, legs of microrobots, and energy harvesting, to mention a few [19,21,22]. We consider four common designs of bimorphs, and examine their performance as actuators and sensors as a function of structural size. 


\section{Computational Model}

We model the electromechanical response of bimorphs using a linear theory of piezoelectricity with poling [23], augmented with flexoelectricity [24]. We summarize next the theory of this model previously presented in Refs. [4,5]. The electrical enthalpy density of a linear dielectric solid possessing piezoelectricity and flexoelectricity can be written as

$$
\begin{aligned}
\mathcal{H}\left(\varepsilon_{i j}, E_{i}, \varepsilon_{j k, l}\right) & =\frac{1}{2} \mathbf{C}_{i j k l} \varepsilon_{i j} \varepsilon_{k l}-e_{i k l} E_{i} \varepsilon_{k l} \\
& -\mu_{i j k l} E_{i} \varepsilon_{j k, l}-\frac{1}{2} k_{i j} E_{i} E_{j},
\end{aligned}
$$

where $\mathbf{E}$ is the electric field, defined as $\mathbf{E}=-\nabla \phi, \phi$ being the electric potential. The first term is the elastic potential, where $\mathbf{C}$ is the fourth-order tensor of elastic moduli. The piezoelectric coupling between strain and electric field is through the second term with the third-order tensor of piezoelectric-

ity e. The last term is the electrostatic potential, where $\mathbf{k}$ is the second-order dielectric tensor. The flexoelectric coupling between the gradient of strain $\nabla \varepsilon$ and the electric field is through the third term, where the flexoelectric tensor $\mu$ represents the combination of direct and converse flexoelectric effects [12]. In this formulation, the remanent state of the piezoelectric material has been taken as the reference configuration, and the poling of the piezoelectric material is implic-

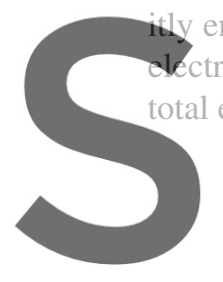
encoded in the constitutive ctric tensor e [23]. In the ab otal electromechanical enthalp is
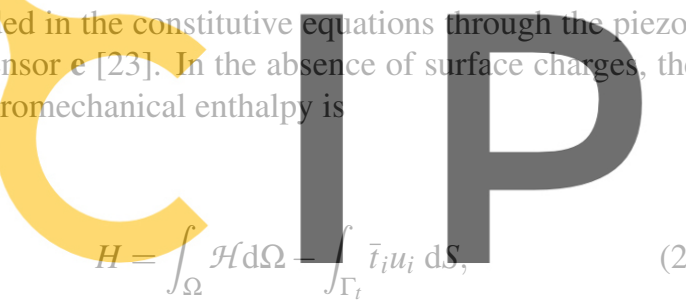

Register for free at https//www.scipedia.com to ary. By making this enthalpy stationary with respect to the displacement and electric potential fields, the self-consistent governing equations in weak form are obtained for the electro-mechanical boundary value problem [4]. Numerically, we deal with the fourth-order nature of the partial differential equations by approximating displacements and electric potential using a meshfree method with smooth basis functions [25]. Unlike our 3D study of flexoelectric effects in pyramids [5], here we restrict out attention to $2 \mathrm{D}$ plane strain conditions for conceptual clarity. The strain gradient elasticity (SGE) term, which guarantees the thermodynamic stability of the system at small scales [26], is not included in our calculations because its effect is very small for flexoelectric multilayer structures of technologically relevant scales [27]. However, while the SGE term has been shown to be insignificant in some situations, it is possible that this term is important in other cases. We checked that its effect was negligible in the simulations reported below. We also note that, while we focus here on bulk flexoelectricity, it has been argued that its phenomenology can be the result of surface piezoelectricity. In this interpretation, surface relaxation induces a local symmetry loss, creating an effectively piezoelectric superficial layer. Its size-dependent effect is
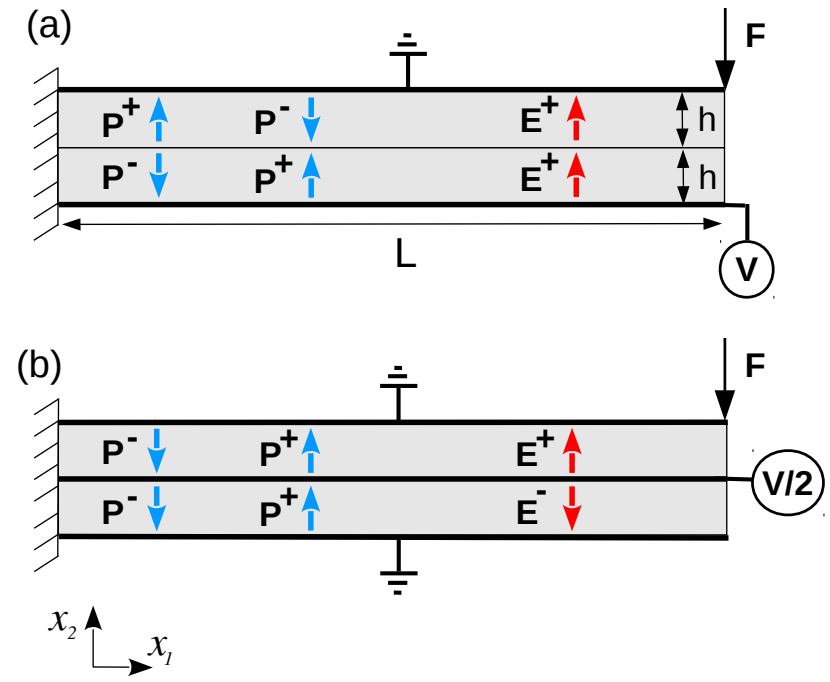

Fig. 1. Piezoelectric bimorph cantilever beams consist of two identical piezoelectric layers (a) series arrangement and (b) parallel arrangement. The beams are mechanically fixed at the left-end and the mechanical point load $F$ is applied at the right-end. In the series bimorph, the electric potential is fixed to zero at the top face while it is fixed to zero at the top and bottom faces of the parallel bimorph. An active electrode is placed at the bottom of the series bimorph and at the layers interface in the parallel bimorph. This active electrode

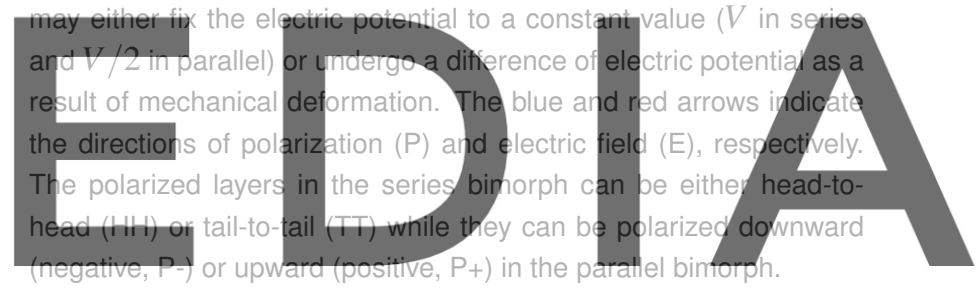

\section{download the version without the watermark} negligible at macroscopic scales, but can be as significant as bulk flexoelectricity at small scales and has the same symmetry [28, 29]. Thus, from a practical viewpoint, surface piezoelectricity and flexoelectricity play the same role.

Piezoelectric bimorphs consist of two identical and perfectly bonded piezoelectric layers, with an electrode configuration such that when an electric field is applied, one layer elongates and the other contracts. This results in a bending deformation of the composite structure. Figure 1 shows the geometry and typical bimorph configurations. Two arrangements are commonly considered. The first type of bimorph shown in Fig. 1(a) is often called series bimorph or antiparallel bimorph. In this arrangement, piezoelectric layers are poled in opposite direction, either head-to-head $(\mathrm{HH})$ or tail-to-tail (TT). The electric potential is applied between the bottom and top faces, generating the same nominal electric field in both layers. The displacement and the electric field are continuous across the interface between the layers in the series device. The second arrangement is known as parallel piezoelectric bimorph and has an intermediate electrode at the interface between the two piezoelectric layers, as depicted in Fig. 1(b). In this case, both layers are poled in the same direction. The polarization can be either downward (negative) or upward (positive). However, the arrangement 


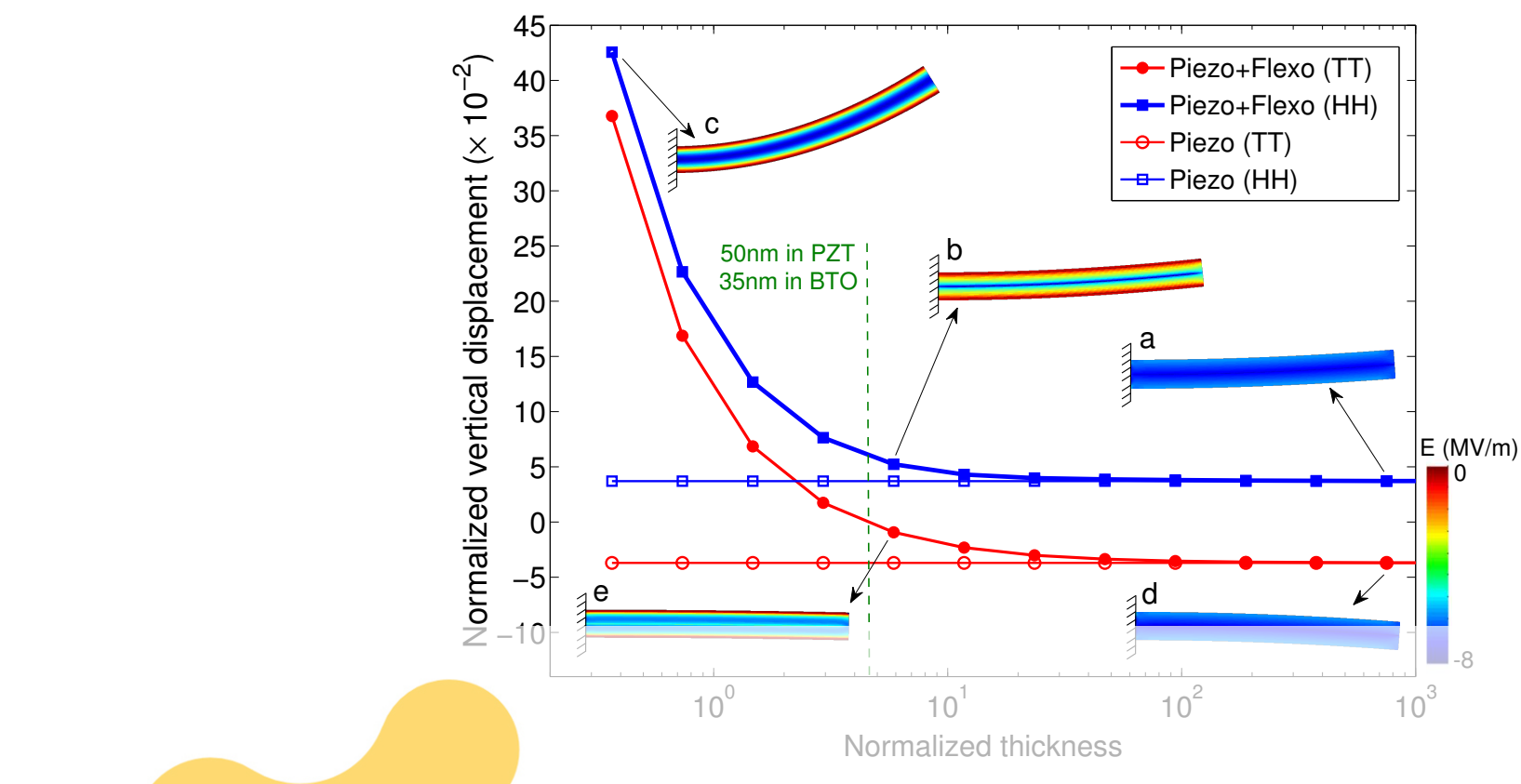

Fig. 2. Normalized vertical displacement as a function of the normalized beam thickness for the series bimorph arrangements: tail-to-tail (TT) and head-to-head (HH) polarized layers. The results are obtained for the piezoelectric bimorphs with and without flexoelectricity. The insets show the distribution of electric field in both arrangements for different beam thicknesses.

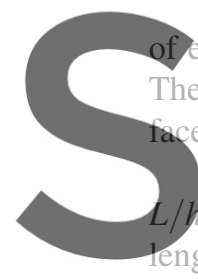

lectrodes results in opposit Therefore, the electric field is d in the parallel device. The aspect ratio of the piezo $=10$ unless otherwise sta ngth and height of the layers.
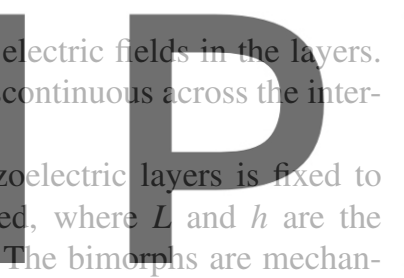
ically constrained at the left-end, and a point force $F$ is ap-

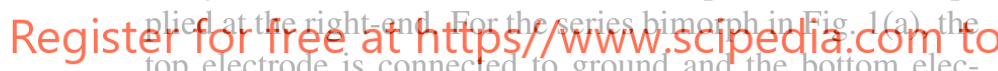
trode is active. For the parallel bimorph in Fig. 1(b), the top and botiom electrodes are connected to ground and the intermediate electrode is active. The active electrode can either prescribe a voltage difference ( $V$ in series and $V / 2$ in parallel), or experience a change of electric potential as a result of deformation. In the latter case, we constrain the electric potential to be constant at the active surface, and find its value as a result of the numerical calculation using Lagrange multipliers. The material parameters are chosen to fit the behavior of PZT-5H [30], a common piezoelectric material for bimorphs. We consider both the longitudinal and transversal flexoelectric coefficients $\mu_{11}$ and $\mu_{12}$. The magnitude of these coefficients is chosen as $\mu=\chi f$, where $f$ is the flexocoupling coefficient and $\chi$ is the dielectric susceptibility of PZT. The value of $f$ has been estimated to be of the order of $1-10 \mathrm{~V}$ for simple ionic solids [3]. Here, we choose $f=10 \mathrm{~V}$.

\section{Bimorph Actuator}

The bimorph structures shown in Fig. 1 exhibit bending deformation upon application of a voltage difference at the electrodes. Using our computational model, we evaluate the actuation vertical displacement of the cantilever tip for dif-
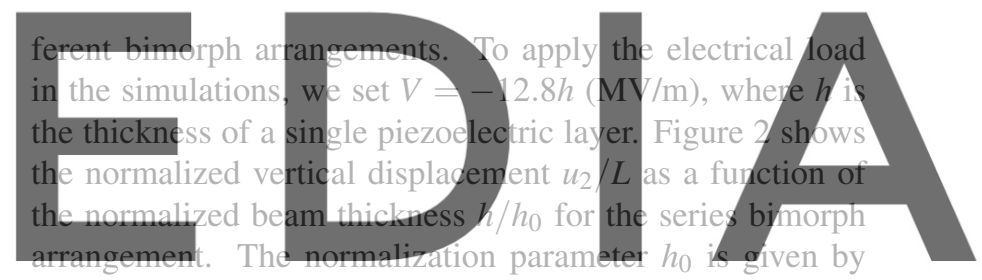

$h_{0}=\mu / e_{33}$, representing a length-scale emanating from the

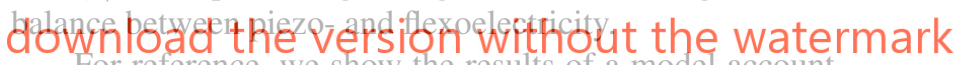
For reference, we show the results of a model accounting for piezoelectricity but not for flexoelectricity. As expected, the response is independent of the size of the beam, and the sign of the displacement changes with polarization reversal (e.g. from head-to-head to tail-to-tail). By accounting for flexoelectricity, however, the actuation response of the beam becomes size-dependent and sensitive to the polarization arrangement of the device. The actuation is considerably enhanced in the head-to-head design, converging to the purely flexoelectric response at the small-scale limit. To investigate the source of this actuation, the distribution of the electric field is shown as insets in Fig. 2 for different beam thicknesses. This distribution is nearly uniform across the thickness for large systems, where the response is dominated by piezoelectricity, see Fig. 2(a). However, by decreasing the bimorph size, the electric field becomes non-uniform across the thickness with considerable gradients near the top and bottom electrodes, c.f. Fig. 2(b) and (c). These gradients, with opposing sign in each of the layers, generate high stresses and an actuation mechanical moment because of converse flexoelectricity, as discussed in detail in Ref. [4]. The flexoelectrically-induced moment always contracts the positive electrode, irrespective of the polarization orientation. In contrast, the mechanical moment induced by piezo- 


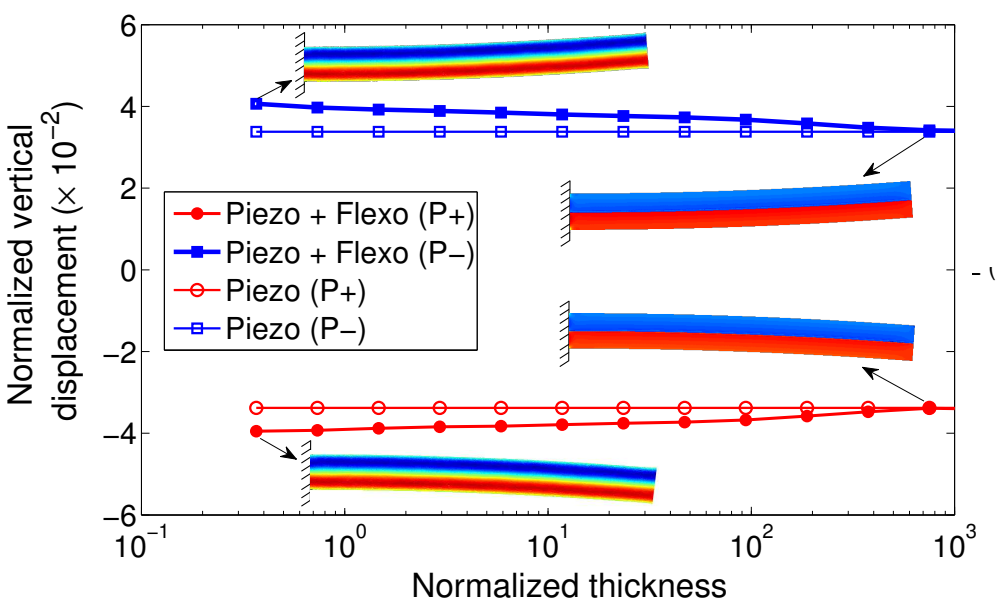

Fig. 3. Normalized vertical displacement as a function of the normalized beam thickness tor the parallel bimorph arrangements: negatively
$(\mathrm{P}$-) and positively $(\mathrm{P}+$ ) polarized layers. The results are obtained for the piezoelectric bimorphs with and withou flexoelectricity. The insets (P.) and positively (P+) polarized layers. The results are obtained for the piezoelectric bim.
show the distribution of electric field in both arrangements tor different beam thicknesses.
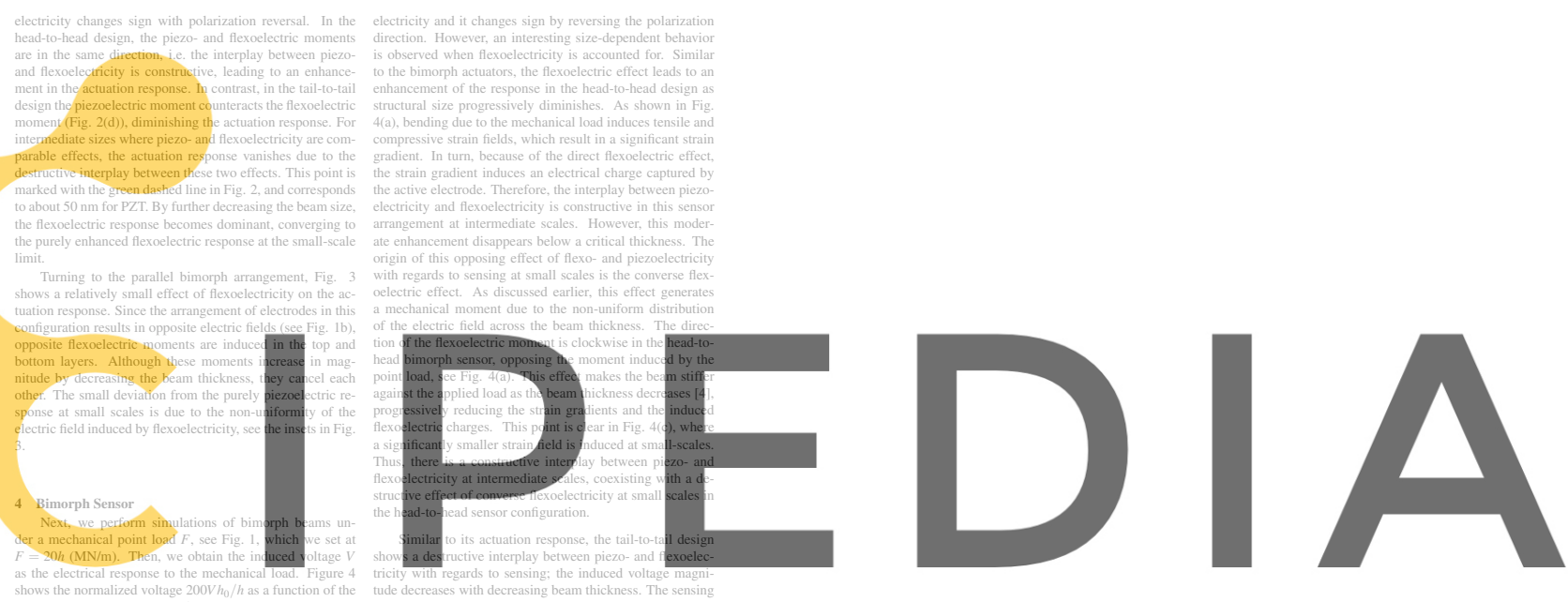

Register for free at https//www.scipedia.com to download the version without the watermark 

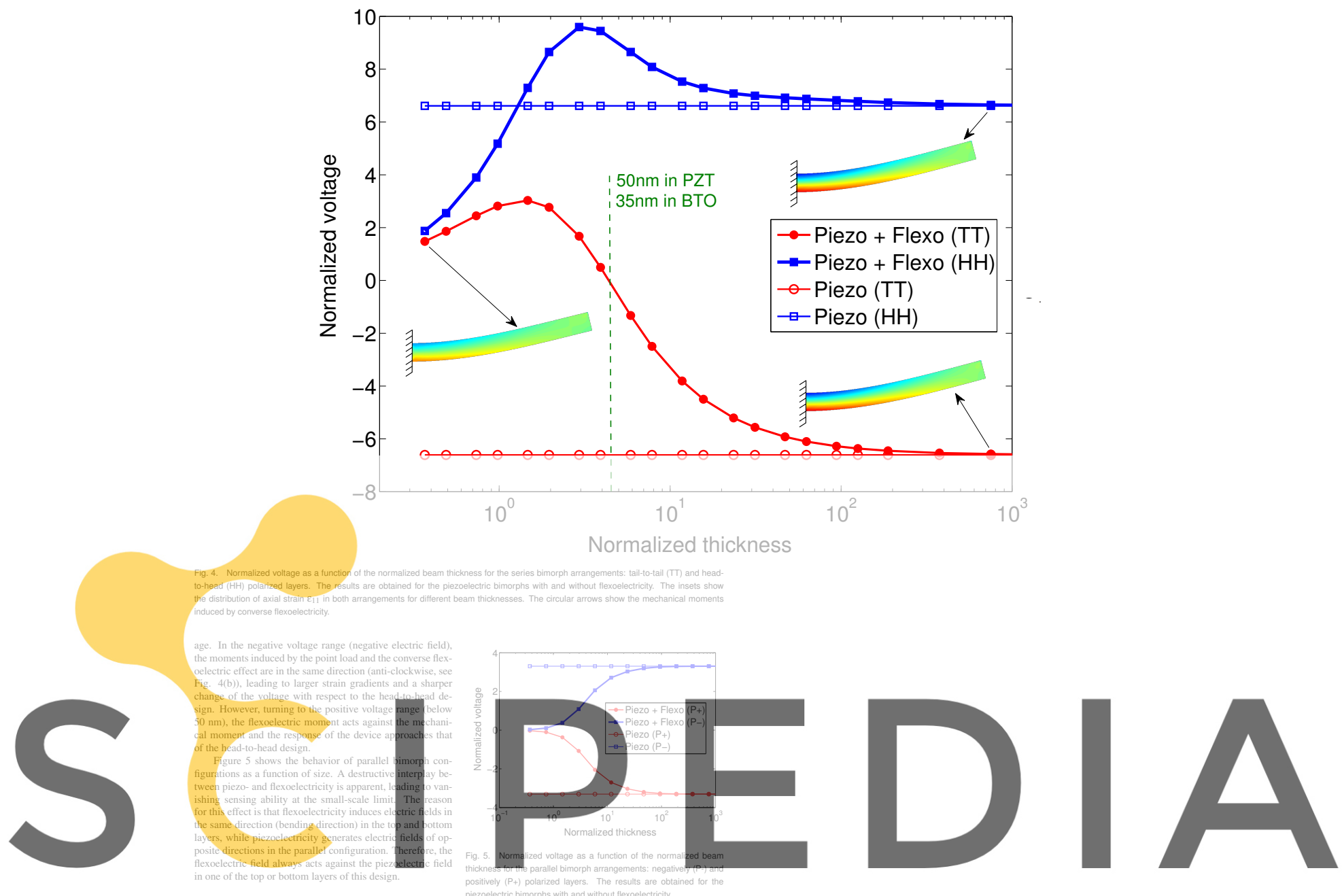

Register for freme and hattps//www. scipedia.corn to download the version without the watermark

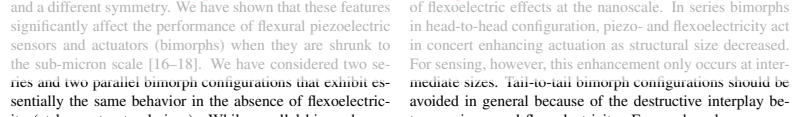

sentially the same behavior in the absence of flexoelectric- avoided in general because of the destructive interplay be-

ity (al large stractire 
tive, our results suggest that flexoelectric-aware designs can significantly enhance the performance of sub-micron piezoelectric transducers, whereas ignoring flexoelectricity can result in degraded performance. In addition to bimorph beams, our results could be extended to atomically thin sheets which exhibit anomalous piezoelectricity due to flexoelectric effects [31].

\section{References}

[1] Yudin, P. V., and Tagantsev, A. K., 2013. "Fundamentals of flexoelectricity in solids". Nanotech., 24(43), p. 432001 .

[2] Nguyen, T. D., Mao, S., Yeh, Y.-W., Purohit, P. K., and McAlpine, M. C., 2013. "Nanoscale flexoelectricity". Adv. Mater., 25(7), pp. 946-974.

[3] Zubko, P., Catalan, G., and Tagantsev, A. K., 2013. "Flexoelectric effect in solids". Annu. Rev. Mater. Res., 43, pp. 387-421.

[4] Abdollahi, A., Peco, C., Millán, D., Arroyo, M., and Arias, I., 2014. "Computational evaluation of the flexoelectric effect in dielectric solids.”. J. Appl. Phys., 116, p. 093502

[5] Abdollahi, A., Millan, D., Peco, C., Arroyo, M., and Arias, I., 2015. "Revisiting pyramid compression to quantify flexoelectricity : a 3 d simulation study". Phys. Rev. B, 91, p. 104103.

[6] Huang, W., Kwon, and Jiang, X., 2013. celerometer". J. Intell. M 277. Kwon, S. R., Huang, W. B., Zhang, S. J., Yuan, F. G. and Jiang, X. N., 2013. "Flexoelectric sensing us-

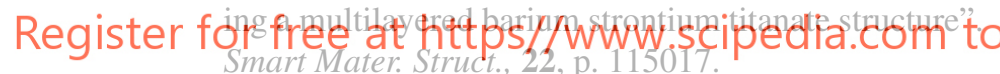
Majdoub, M. S., Sharma, P., and Cagin, T., 2008. "Dramatic enhancement in energy harvesting for a narrow range of dimensions in piezoelectric nanostructures". Phys. Rev. B, 78, p. 121407.

[9] Qi, Y., Kim, J., Nguyen, T. D., Lisko, B., Purohit, P. K., and McAlpine, M. C., 2011. "Enhanced piezoelectricity and stretchability in energy harvesting devices fabricated from buckled PZT ribbons". Nano Lett., 11(3), pp. 1331-1336.

[10] Deng, Q., Kammoun, M., Erturk, A., and Sharma, P., 2014. "Nanoscale flexoelectric energy harvesting". Int. J. Solids Struct., 51(18), pp. 3218-3225.

[11] Sharma, N. D., Maranganti, R., and Sharma, P., 2007. "On the possibility of piezoelectric nanocomposites without using piezoelectric materials". J. Mech. Phys. Solids, 55(11), pp. 2328-2350.

[12] Sharma, N. D., Landis, C. M., and Sharma, P., 2010. "Piezoelectric thin-film superlattices without using piezoelectric materials". J. Appl. Phys., 108(2), p. 024304.

[13] Deng, Q., Liu, L., and Sharma, P., 2014. "Electrets in soft materials: Nonlinearity, size effects, and giant electromechanical coupling”. Phys. Rev. E, 90.
[14] Deng, Q., Liu, L., and Sharma, P., 2014. "Flexoelectricity in soft materials and biological membranes". $J$. Mech. Phys. Solids, 62, pp. 209-227.

[15] Mbarki, R., Baccam, N., Dayal, K., and Sharma, P., 2014. "Piezoelectricity above the curie temperature? combining flexoelectricity and functional grading to enable high-temperature electromechanical coupling". Appl. Phys. Lett., 104, p. 122904.

[16] Ivaldi, P., Abergel, J., Matheny, M. H., Villanueva, L. G., Karabalin, R. B., Roukes, M. L., Andreucci, P., Hentz, S., and Defaÿ, E., 2011. "50 nm thick AlN film-based piezoelectric cantilevers for gravimetric detection". J. Micromech. Microeng., 21, p. 085023.

[17] Sinha, N., Wabiszewski, G. E., Mahameed, R., Felmetsger, V. V., Tanner, S. M., Carpick, R. W., and Piazza, G., 2009. "Piezoelectric aluminum nitride nanoelectromechanical actuators". Appl. Phys. Lett., 95, p. 053106

[18] Kang, S. J., Park, Y. J., Bae, I., Kim, K. J., Kim, H. C., Bauer, S., Thomas, E. L., and Park, C., 2009. "Printable ferroelectric PVDF/PMMA blend films with ultralow roughness for low voltage non-volatile polymer memory". Adv. Funct. Mater., 19, pp. 2812-2818.

[19] Smits, J. G., Dalke, S. I., and Cooney, T. K., 1991. "The constituent equations of piezoelectric bimorphs". Sens.

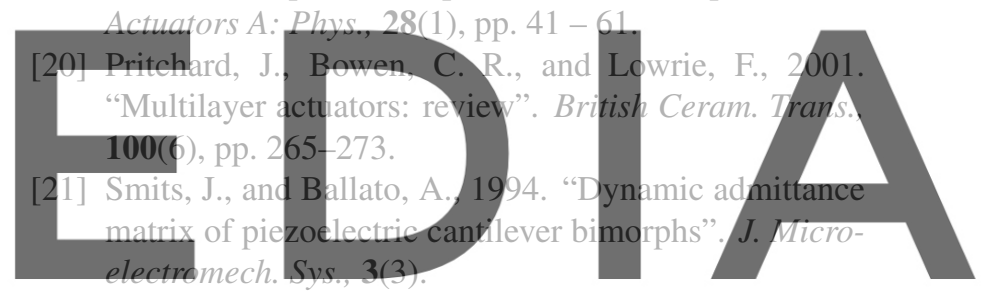

[22] Sodano, H., Inman, D., and Park, G., 2004. "A review

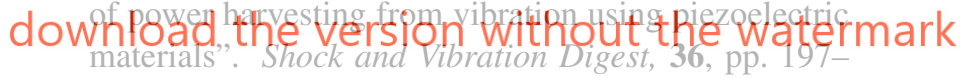
205

[23] Abdollahi, A., and Arias, 1., 2012. "Phase-field mode1ing of crack propagation in piezoelectric and ferroelectric materials with different electro-mechanical crack conditions". J. Mech. Phys. Solids, 60, p. 2100.

[24] Catalan, G., Sinnamon, L. J., and Gregg, J. M., 2004. "The effect of flexoelectricity on the dielectric properties of inhomogeneously strained ferroelectric thin films". J. Phys.: Cond. Matter, 16(13), p. 2253.

[25] Arroyo, M., and Ortiz, M., 2006. "Local maximumentropy approximation schemes: a seamless bridge between finite elements and meshfree methods". Int. J. Numer. Meth. Eng., 65(13), pp. 2167-2202.

[26] Mao, S., and Purohit, P. K., 2014. "Insights into flexoelectric solids from strain-gradient elasticity". J. Appl. Mech., 81, p. 081004.

[27] Sharma, N. D., Landis, C., and Sharma, P., 2012. "Erratum: Piezoelectric thin-film super-lattices without using piezoelectric materials [j. appl. phys. 108, 024304 (2010)]". J. Appl. Phys., 111(5), p. 059901.

[28] Tagantsev, A., and Yurkov, A., 2012. "Flexoelectric effect in finite samples". J. Appl. Phys., 112(4), p. 044103. 
[29] Stengel, M., 2013. "Microscopic response to inhomogeneous deformations in curvilinear coordinates". $\mathrm{Na}$ ture Commun., 4, p. 2693.

[30] Zhu, S., Jiang, B., and Cao, W., 1998. "Characterization of piezoelectric materials using ultrasonic and resonant techniques". Proc. SPIE, 3341, pp. 154-162.

[31] Zelisko, M., Hanlumyuang, Y., Yang, S., Liu, Y., Lei, C., Li, J., Ajayan, P., and Sharma, P., 2014. "Anomalous piezoelectricity in two-dimensional graphene nitride nanosheets". Nature Commun., 5, p. 4284.

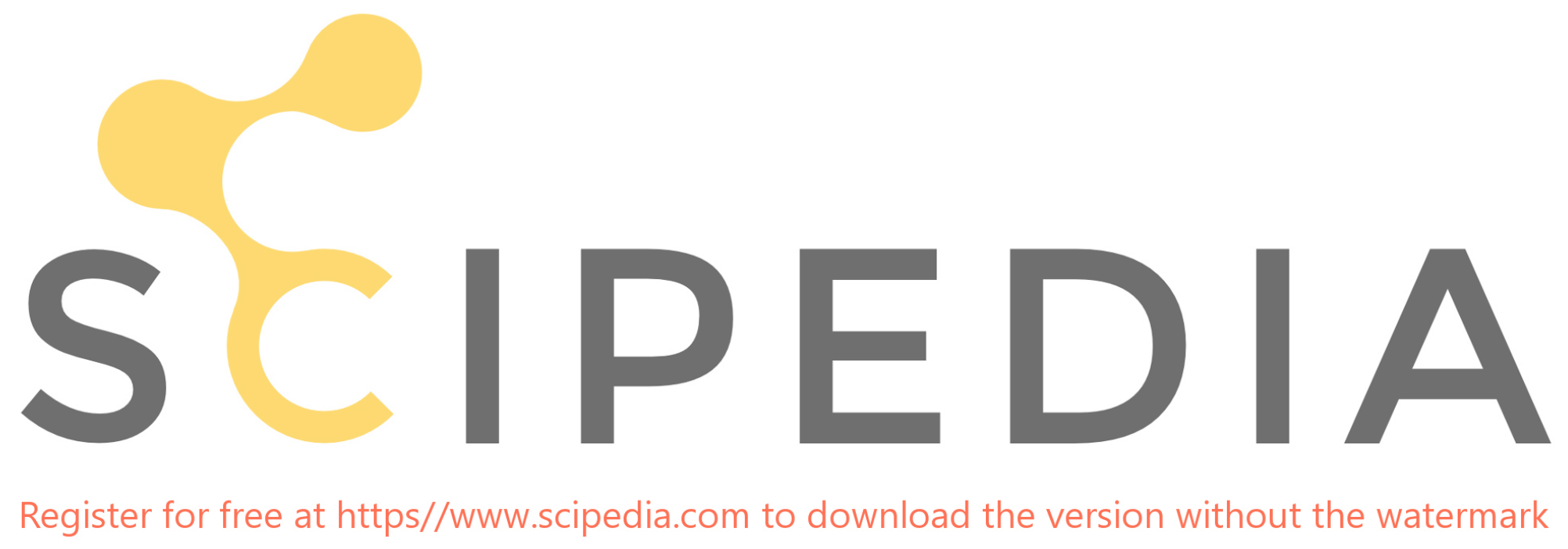

